\title{
Comparison of In Vitro Dissolution Tests for Commercially Available Aspirin Tablets
}

\author{
Fawzia Khan, Mingzhong Li*, and Walkiria Schlindwein \\ School of Pharmacy, De Montfort University, the Gateway, Leicester, LE1 9BH, UK
}

\begin{abstract}
In this work, quality control tests were carried out to evaluate several quality characteristics of five brands of commercially available aspirin tablets including mechanical hardness, friability, disintegration, and in vitro dissolution. Brands $\mathrm{A}$ and $\mathrm{B}$ or brands C, D, and E had similar results for hardness, friability, and disintegration rates, indicating that the two groups of the brands had different manufacturing conditions. Comparisons of dissolution profiles of the different brands were carried out by both model-independent and model-dependent approaches. Results obtained via the model-dependent approach show a first-order drug release mechanism for all five brands of aspirin tablets. From both difference and similarity factors, it was concluded that all five brands of commercially available aspirin tablets are similar.
\end{abstract}

\section{INTRODUCTION}

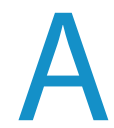
spirin is one of the most commonly used drugs in the world with approximately 35,000 tons produced and consumed annually (1). Aspirin is a nonsteroidal anti-inflammatory drug (NSAID) used for treating fever, pain, and inflammation in the body; its chemical structure is shown in Figure 1. NSAIDs work by reducing the levels of prostaglandins released when there is inflammation that causes pain and fever. The inhibition of prostaglandins also reduces the function of platelets and the ability of blood to clot with effects lasting longer than other NSAIDs (2). Recognition of this antiplatelet activity has led to the emergence of aspirin as an important drug in the management of cardiovascular and cerebrovascular disease, particularly in preventing heart attacks and strokes (3). There is also increasing evidence that aspirin reduces the risk of some cancers (4). Currently several generic aspirin drugs formulated as immediaterelease tablets are available over the counter under different brands. Because of this, it would be very important to investigate the potential for differences among different brands of aspirin, as various factors such as formulation and manufacturing process may affect the therapeutic effects of the drug.

Dissolution tests are widely used in the pharmaceutical industry for developing new drug products (5), determining the long-term stability and shelf life of a dosage form (6), and assessing the impact of post-approval changes in the manufacturing process. Dissolution tests can be prognostic of in vivo performance of drug products if the conditions in the gastrointestinal tract are reconstructed successfully in the experiments. Many regulatory authorities such as the FDA have introduced guidelines on dissolution testing of solid dosage forms for the pharmaceutical industry (6). In the case of immediate-release solid dosage forms such as tablets, dissolution tests are

${ }^{*}$ Corresponding author. used to evaluate batches and can be used as a guide to develop new and existing formulations and possibly to assess the impact of certain changes in the formulation and manufacturing processes. Dissolution tests can also provide the ability to discriminate between interbatch discrepancies within a product and provide a specification for in vitro drug release allowing determination of in vivo suitability of the formulation (7). The evaluation of in vitro equivalence between reference and test batches can be conducted by the use of dissolution profile comparison and analysis. Very often, an in vitro dissolution test is more sensitive and discriminating than an in vivo test. From a quality assurance point of view, a more discriminative dissolution method is preferred, because the test will indicate possible changes in the quality of the product before in vivo performance is affected (7).

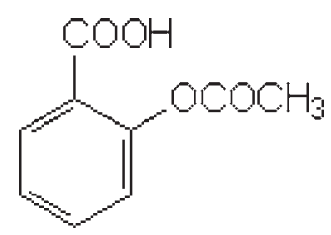

Figure 1. Structure of aspirin (acetylsalicylic acid, 2-acetyloxybenzoic acid).

Methods for comparing in vitro dissolution profiles can be classified into three main groups: ANOVA-based statistical methods, model-independent and modeldependent approaches. ANOVA-based methods can be classified as one-way analysis of variance (ANOVA) and multivariate analysis of variance (MANOVA), which assess the difference between the means of two drug release data sets at a single time point of dissolution and at multiple time points of dissolution, respectively. Many studies have shown that the ANOVA-based methods were overly discriminating and that it was difficult to distin- 
guish between two dissolution curves (8-10). The modelindependent approach allows the profile or profile differences to be translated into a single value enabling a simpler analysis of data (9-18). Two model-independent techniques are commonly used, ratio tests and fit factors. The most common ratio tests include comparison of the mean dissolution times (MDTs), variance of dissolution times (VTs), and the relative dispersion of dissolution times (RDs). Although the ratio test approaches are not mentioned in FDA guidance documents (7), they are very useful statistical tools for identifying similarities in the data and, in particular, for establishing the correlation between in vitro and in vivo performance (19); the techniques are also easy to apply. Fit factors, also known as the difference factor $f_{1}$ and the similarity factor $f_{2}$, were introduced by Moore and Flanner (17). The factor $f_{1}$ is a measure of the average percentage difference of drug released over selected time points between the dissolution profiles. A calculated value of zero for $f_{1}$ indicates that the profiles of the test and reference are identical. As the value increases from zero, dissimilarity between the two profiles increases, and usually a value of 15 or below suggests fair similarity. The $f_{2}$ values are between 0 and 100 , and if the calculated value is greater than 50 , the test and reference formulations are considered similar. This value of greater than 50 was empirically derived assuming that at any sample point, the average difference of percentage release of active drug cannot be more than $10 \%$ (17); however, this assumption may not be true in some cases. The similarity factor $f_{2}$ is gaining popularity due to its recommendation by a number of regulatory authorities as a criterion for the assessment of similarity between dissolution profiles $(11,20,21)$. Modelindependent approaches directly compare the dissolution data without having to rely on model functions that may prove to be artificial. Mathematical models have been used extensively for the representation of dissolution data $(9-11,13,21)$. The approach requires a suitable mathematical function that can be linear or nonlinear to describe the dissolution data. Nonlinear models tend to be more reliable as they predict responses outside the observed range of data, whereas linear models are linear in their parameters (14-15). Some common models of both linear and nonlinear functions include Higuchi, Hixson-Crowell, Korsmeyer-Peppas, Weibull, Logistic, zero-order, and first-order (21). Once a model is selected, the dissolution profiles are compared and evaluated in terms of the model parameters. In the meantime, the mathematical model approach also provides an insight into the drug release mechanism. Although such mathematical models have been used to characterize dissolution profiles, such methods are quite complicated and require caution in their application compared with model-independent methods (10).

The aim of this work was to investigate if there are any differences between various commercially available aspirin tablets through evaluation of in vitro dissolution profiles using both model-dependent and model-independent approaches. Five different brands of immediate-release solid dosage form aspirin tablets were obtained from commercial vendors for this study. Dissolution testing was carried out using USP Apparatus 2 (paddle) to obtain dissolution profiles of aspirin tablets in $900 \mathrm{~mL} \mathrm{pH} 4.5$ acetate buffer with samples taken at 10, $20,30,45,60$, and $90 \mathrm{~min}$. Additional quality control tests were also carried out on each brand of aspirin tablets, including disintegration, friability, and tablet hardness, to provide possible reasons for differences in dissolution profiles.

\section{MATERIALS AND METHODS \\ Materials}

In this study, five brands of commercially available uncoated aspirin tablets were obtained from vendors $A$, $B, C, D$, and E. Detailed information of the different brands of tablets is summarized in Table 1. The labeled amount of drug substance for each brand is the same $(300 \mathrm{mg})$, as are all of the excipients in the formulations of the different brands. However, potato starch was used in brands C, D, and $E$, whereas there is no detailed information about the starch used in brands $A$ and $B$.

Acetate buffer at $\mathrm{pH} 4.5$ was used as the dissolution medium as specified in the British Pharmacopoeia (22). It was prepared by mixing $29.9 \mathrm{~g}$ of sodium acetate with $16.6 \mathrm{~mL}$ of glacial acetic acid and sufficient distilled water to produce $10 \mathrm{~L}$. Sodium acetate and glacial acetic acid were analytical grade and purchased from Sigma-Aldrich UK. Pure aspirin powder (Acetylsalicylic Acid BP, SigmaAldrich UK) was dissolved in the acetate buffer to make a series of standard calibration solutions with different concentrations for development of a calibration curve using a UV spectrophotometer at $265 \mathrm{~nm}$.

Table 1. Aspirin Tablets Used in the Tests

\begin{tabular}{cclll}
\hline Brand & Dosage (mg) & Excipients & Appearance \\
\hline A, Reference & 300 & aspirin, starch, lactose, talc & White, circular with indented line in center \\
\hline B & 300 & aspirin, starch, lactose, talc & White, circular with indented line in center \\
\hline C & 300 & aspirin, (potato) starch, lactose, talc & White, circular with indented line in center \\
\hline D & 300 & aspirin, (potato) starch, lactose, talc & White, circular with indented line in center \\
\hline E & 300 & aspirin, (potato) starch, lactose, talc & White, circular with indented line in center \\
\hline
\end{tabular}




\section{Dissolution Testing}

In vitro dissolution was carried out via USP Apparatus 2 (paddle) at a speed of $75 \mathrm{rpm}$ in $900 \mathrm{~mL}$ of dissolution medium ( $\mathrm{pH} 4.5$ acetate buffer) maintained at $37 \pm 0.5^{\circ} \mathrm{C}$ using a water bath fitted with a variablespeed stirrer and heater (Erweka DT6). Selection of 75rpm rotation speed was based on the British Pharmacopoeia guideline (22). Samples $(5 \pm 0.1 \mathrm{~mL})$ were taken manually at 10,20,30, 45, 60, and 90 min and replaced with an equal volume of fresh medium to maintain a constant dissolution volume. The samples were filtered, and the absorbance was measured at $265 \mathrm{~nm}$ using a UV spectrophotometer (PU 8625 UV/VIS spectrophotometer). The drug concentration determined by the calibration model was used to calculate the total mass of the drug released in the medium. In this work, the dissolution profiles are represented as the cumulative percentages of the amount of drug released at each sampling interval. Each profile is the average of six individual tablets.

\section{Tablet Disintegration, Friability, and Hardness Tests}

The disintegration test was carried out as stated in the British Pharmacopoeia (22), in which one dosage unit was placed in each of the six tubes of the basket with a tablet on the top of the disc (Pharma Test DIST 3). Acetate buffer at $\mathrm{pH} 4.5$ was used as the immersion fluid at $37^{\circ} \mathrm{C}$. The specification states that all of the uncoated tablets should disintegrate within $15 \mathrm{~min}$ (22). The end point was determined when there were no particles or granules remaining on the disc.

The friability test method was adapted from that in the British Pharmacopoeia (22). The BP method states that 20 tablets are rotated in the friability drum (Pharma Test PTF E) 100 times. As there was a limited supply of aspirin tablets in this study, the quantity was halved to 10 tablets.

In the hardness test, six tablets were inserted individually into the tablet crushing machine (C40 Tablet Crusher, Engineering Systems Nottm Ltd.) lined up with the center of the vice. When the device was started, the vice gradually applied force onto the tablet until it split, and the force at which this occurred was recorded.

\section{Analysis and Comparison of Dissolution Profiles}

In this study, both model-independent and modeldependent methods were selected to compare different dissolution profiles.

\section{Model-Independent Methods}

Model-independent approaches produce a single value from a dissolution profile, providing direct comparisons of the dissolution data. Consequently the results do not depend on the selection of the specific parameter for fitting data but on the chosen sampling time $t_{1}(i=1, \ldots, n)$ in the calculation. Model-independent approaches include ratio tests and fit factors.

\section{Ratio-Test Approaches}

Ratio tests are performed as ratios of percent drug dissolved, area under the dissolution curve, and mean dissolution times of the reference formulation with those of a test formulation at the same sampling time. The most common ratio test is performed by comparison of two mean dissolution times (MDTs), which are calculated by

$$
\text { MDT }=\frac{\sum_{i}^{n} t_{i} \Delta M_{i}}{\sum_{i}^{n} \Delta M_{i}}
$$

where $i$ is the sample number, $n$ is the number of dissolution sample times, $\bar{t}_{i}=\frac{t_{i-1}+t_{i}}{2}$ is the time at midpoint between $t_{i-1}$ and $t_{i}$, and $\Delta M_{i}$ is the additional amount of drug dissolved between $t_{i-1}$ and $t_{i}$.

The variance of dissolution times (VTs) is estimated by

$$
V T=\frac{\sum_{i}^{n}\left(\overline{t_{i}}-M D T\right)^{2} \Delta M_{i}}{\sum_{i}^{n} \Delta M_{i}}
$$

and the relative dispersion of dissolution times (RD) is given by

$$
\mathrm{RD}=\frac{\mathrm{VT}}{\mathrm{MDT}^{2}}
$$

\section{Fit Factors}

Fit factors include a difference factor $f_{1}$ and a similarity factor $f_{2}$. The difference factor $f_{1}$ calculates the percentage difference between the two curves at each point and measures the percentage error between them over all time points, which is given by

$$
\mathrm{f}_{1}=\frac{\sum_{\mathrm{i}=1}^{\mathrm{n}}\left|\mathrm{R}_{\mathrm{i}}-\mathrm{T}_{\mathrm{i}}\right|}{\sum_{\mathrm{i}=1}^{\mathrm{n}} \mathrm{R}_{\mathrm{i}}}
$$

where $R_{i}$ and $T_{i}$ are the percent drug dissolved of the reference and test products, respectively, at each sample point i.

The similarity factor $f_{2}$ is a logarithmic reciprocal square root transformation of the sum of squared error of differences between the test and product dissolution profiles over all time points and is a measurement of the similarity between the curves, which is given by

$$
f_{2}=50 \log \left\{\left[1+\frac{1}{n} \sum_{i=1}^{n}\left(R_{i}-T_{i}\right)^{2}\right]^{-0.5} \times 100\right\}
$$

\section{Model-Dependent Methods}

Different mathematical models have been proposed to analyze the dissolution profiles through which the mechanism of drug release can be determined. The statistical comparison of the dissolution profiles can be performed by a $t$-test for the estimated parameters of 
the regression functions (9-11). The mathematical models of a dissolution profile can be deduced by a theoretical analysis of the process, such as zero-order kinetics, first-order kinetics, Higuchi, and Hixson-Crowell models. In most cases, due to the complexity of dosage forms (e.g., tablets, capsules, coated or prolonged-release dosage forms), a theoretical foundation does not exist; as a result, semi-empirical or empirical models (e.g., Korsmeyer-Peppas, Weibull, and logistic models) have to be used to fit dissolution data. The most common models in the dissolution data analysis are summarized in Table 2 (11). In general, the final sample time is selected when each individual profile reaches at least $80 \%$. The mathematical models were fitted to six individual dissolution data of each brand of aspirin tablets using the Statistics Toolbox module of Matlab 6.5. The model parameters were determined by the Gauss-Newton algorithm with Levenberg-Marquardt modifications for global convergence. The statistical mean and standard deviation of the parameters of mathematical models for each brand can be obtained based on the calculation results. The model parameters of the dissolution profiles of the different brands of aspirin tablets were compared with the reference using a two-variable $t$-test as follows:

$$
t=\frac{\left|\overline{x_{R}}-\overline{x_{T}}\right|}{S_{d}} \sqrt{\frac{n_{R} n_{T}}{n_{R}+n_{T}}}
$$

where $\overline{x_{R}}$ and $\overline{x_{T}}$ are means of the model parameters of the reference and test products, respectively, $n_{R}$ and $n_{T}$ are the numbers of measurements for the mean $\overline{x_{R}}$ and
$\overline{\mathrm{x}_{\mathrm{T}}}$, and $\mathrm{S}_{d}$ is the weighted average standard deviation as shown below

$$
S_{d}=\sqrt{\frac{\left(n_{R}-1\right) s_{R}^{2}+\left(n_{T}-1\right) s_{T}^{2}}{n_{R}+n_{T}-2}}
$$

where $S_{R}$ and $S_{T}$ are the standard deviations of the model parameters for the reference and test products.

If the calculated $t$ value is less than the critical value of $t\left(1-\frac{\alpha}{2} ; f=n_{1}+n_{2}-2\right)$, the two means $\overline{\mathrm{x}_{\mathrm{R}}}$ and $\overline{\mathrm{x}_{\mathrm{T}}}$ differ only randomly at risk level $\alpha$.

\section{RESULTS AND DISCUSSION \\ Tablet Disintegration, Friability, and Hardness Tests}

The average disintegration times of six aspirin tablets for each brand are shown in Table 3. The results show that all of the tablets disintegrated quickly (within $2 \mathrm{~min}$ ) thereby complying with the $B P$ specification in which six immediate-release tablets must disintegrate within 15 min using a standard disintegration apparatus (22). Brands A and B have similar disintegration times, whereas those of brands $C, D$, and $E$ are almost equal. Brands $A$ and $B$ tablets took twice as long to disintegrate as those of brands $C, D$, and $E$.

Ten tablets of each brand were used in the friability tests, and weight losses of the different brands of tablets are shown in Table 4. All of brands of tablets comply with the $B P$ specifications, which state that the maximum loss in weight must not exceed $1 \%$ of the original mass (22). Brands $A$ and $B$ or brands $C$ and $D$ are similar, whereas the weight loss of brand $E$ is almost twice that of brands $A$ and $B$.

\begin{tabular}{|c|c|c|c|c|c|}
\hline Model & & Mathematical equation & Release mechanism & Theoretical RD ${ }^{a}$ & Release class \\
\hline Zero-order & & $c_{1} t$ & Constant release rate & 0.3 & 0 \\
\hline First-order & & $c_{2}\left(1-\exp \left(-c_{1}\right)\right)$ & Fick's first law diffusion mechanism & 1.0 & 1 \\
\hline Higuchi & & $c_{1} t^{0.5}$ & Diffusion medium based mechanism & 0.8 & 2 \\
\hline Hixson-Crowell & & $c_{2}\left(1-\left(1-c_{1} t\right)^{3}\right)$ & Erosion release mechanism & 0.6 & 3 \\
\hline Korsmeyer-Peppas & & $c_{2} t^{c_{1}}$ & $\begin{array}{l}\text { Semi-empirical model, diffusion medium } \\
\text { based mechanism }\end{array}$ & & \\
\hline Weibull & & $c_{3}\left(1-\exp \left(-\frac{t}{c_{1}}\right) c_{2}\right)$ & $\begin{array}{l}\text { Empirical model, lifetime distribution } \\
\text { function }\end{array}$ & & \\
\hline \multirow{2}{*}{ Logistic } & \multirow[t]{2}{*}{$c_{3}$} & $\exp \left(c_{1}+c_{2} \log t\right)$ & \multirow{2}{*}{ Empirical model, S-shaped model } & & \\
\hline & & $1+\exp \left(c_{1}+c_{2} \log t\right)$ & & & \\
\hline
\end{tabular}

Table 2. Mathematical Models for Drug Dissolution Profile Analysis

${ }^{a}$ Relative dispersion of dissolution time 
Table 3. Disintegration Times of the Five Brands of Aspirin Tablets in pH 4.5 Acetate Buffer at $37^{\circ} \mathrm{C}$

\begin{tabular}{cc}
\hline Brand & Disintegration Time (sec) \\
\hline A & 93 \\
\hline B & 100 \\
\hline C & 53 \\
\hline D & 49 \\
\hline E & 56 \\
\hline
\end{tabular}

Six tablets of each brand were used in the hardness tests and the average crushing strength for each brand is shown in Table 5. It is shown that the brand $A$ and $B$ tablets require the greatest force to break them whereas tablets $C, D$, and $E$ require approximately only a third of the force of brand $A$ and $B$ tablets for them to break.

From the test results above, it is clear that the five brands of aspirin tablets can be classified into two groups, brands $A$ and $B$ or brands $C, D$, and $E$ tablets, in which both groups have similar physical properties. It is known that the compression force during a tableting process plays an essential role on the overall properties of the products, such as tablet disintegration rate, friability, and hardness (23). It is therefore concluded that the compression forces used during the manufacturing processes for brands $\mathrm{A}$ and $B$ are likely to be significantly higher than those used for brands $C, D$, and $E$.

\section{In Vitro Dissolution Tests}

Results for the in vitro dissolution tests of five brands of commercially available aspirin tablets are presented in Figure 2 as the average percent drug dissolved versus time. The dissolution data and their statistical properties are given in Table 6. All drugs complied with the dissolution specification stated in the BP that after $45 \mathrm{~min}, 85 \%$ of the drug should be released (22). The profiles in Figure 2 exhibit four stages of dissolution. The greatest percentage of drug dissolved in the shortest time (i.e., approximately $70 \%$ of the drug dissolved within $10 \mathrm{~min}$ ) was at the initial step. Between 10 and $20 \mathrm{~min}$, approximately $15 \%$ more of the drug dissolved, therefore the total percentage of drug dis-
Table 5. Tablet Hardness Results

\begin{tabular}{cc}
\hline Brand & Crushing Strength $\left(\mathbf{k g} / \mathbf{m}^{2}\right)$ \\
\hline A & 11.48 \\
\hline B & 11.04 \\
\hline C & 4.98 \\
\hline D & 4.71 \\
\hline E & 4.49 \\
\hline
\end{tabular}

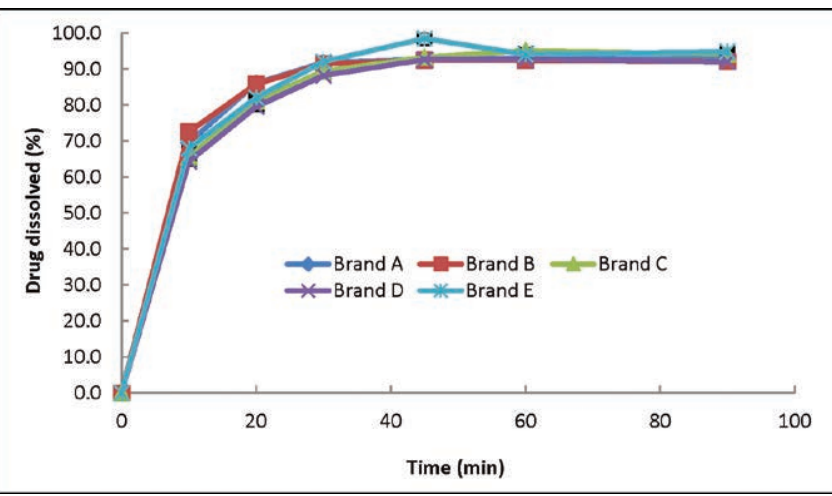

Figure 2. Average $(n=6)$ percent drug dissolved in dissolution of five brands of aspirin tablets in $900 \mathrm{~mL} \mathrm{pH} 4.5$ acetate buffer at $37^{\circ} \mathrm{C}$ for $90 \mathrm{~min}$.

solved at this time is around $85 \%$. The third step between 20 and 30 min shows a further $10 \%$ of drug dissolved. In general, the profiles reached a peak at $45 \mathrm{~min}$ where there is the highest percentage of dissolved drug (around 95\%). After $45 \mathrm{~min}$, the graphs level off to a plateau up to $90 \mathrm{~min}$ with very little change in percentage of drug dissolved. It was surprising that none of the brands was $100 \%$ dissolved. The profiles in Figure 2 clearly show that brand $B$ has a very similar dissolution profile to the reference brand A. Quantitative analyses of the dissolution profiles have been carried out using both the model-independent and model-dependent methods described above.

\section{Model-Independent Methods}

Ratio test parameters of mean dissolution time (MDT), variance of dissolution time (VR), and relative dispersion of dissolution time (RD) for each brand of aspirin tablets

Table 4. Friability Test Results of the Five Brands of Aspirin Tablets

\begin{tabular}{ccccc}
\hline Brand & Weight before (g) & Weight after (g) & Weight loss (g) & Weight loss (\%) \\
\hline A & 3.4438 & 3.4371 & 0.0067 & 0.19 \\
\hline B & 3.4300 & 3.4240 & 0.0060 & 0.17 \\
\hline C & 3.4351 & 3.4261 & 0.0090 & 0.26 \\
\hline D & 3.4615 & 3.4529 & 0.0086 & 0.25 \\
\hline E & 3.4548 & 3.4424 & 0.0124 & 0.36 \\
\hline
\end{tabular}


Table 6. Dissolution Data and Descriptive Statistics of Five Brands of Aspirin Tablets

\begin{tabular}{|c|c|c|c|c|c|c|c|}
\hline Time (min) & Brand & mean \% & SD & SE & $95 \% \mathrm{Cl}$ & Lower limit & Upper limit \\
\hline & A & 69.59 & 2.59 & 1.06 & 2.71 & 66.88 & 72.31 \\
\hline & B & 72.51 & 2.22 & 0.91 & 2.33 & 70.17 & 74.84 \\
\hline \multirow[t]{5}{*}{10} & C & 65.57 & 3.65 & 1.49 & 3.83 & 61.73 & 69.40 \\
\hline & $\mathrm{D}$ & 64.33 & 2.92 & 1.19 & 3.06 & 61.26 & 67.39 \\
\hline & $\mathrm{E}$ & 68.02 & 1.88 & 0.77 & 1.97 & 66.05 & 69.99 \\
\hline & A & 86.12 & 2.05 & 0.84 & 2.15 & 83.96 & 88.27 \\
\hline & $B$ & 85.86 & 1.70 & 0.69 & 1.78 & 84.08 & 87.64 \\
\hline \multirow[t]{5}{*}{20} & C & 81.05 & 4.52 & 1.85 & 4.75 & 76.30 & 85.80 \\
\hline & $D$ & 79.58 & 2.81 & 1.15 & 2.95 & 76.63 & 82.53 \\
\hline & $E$ & 82.07 & 1.26 & 0.51 & 1.32 & 80.74 & 83.39 \\
\hline & A & 91.84 & 1.13 & 0.46 & 1.18 & 90.66 & 93.03 \\
\hline & B & 91.49 & 1.05 & 0.43 & 1.10 & 90.39 & 92.60 \\
\hline \multirow[t]{5}{*}{30} & C & 89.44 & 3.06 & 1.25 & 3.22 & 86.22 & 92.65 \\
\hline & $D$ & 88.23 & 2.11 & 0.86 & 2.22 & 86.01 & 90.45 \\
\hline & $E$ & 92.08 & 0.88 & 0.36 & 0.92 & 91.16 & 93.01 \\
\hline & A & 92.75 & 0.81 & 0.33 & 0.85 & 91.90 & 93.59 \\
\hline & B & 92.50 & 1.18 & 0.48 & 1.23 & 91.27 & 93.74 \\
\hline \multirow[t]{5}{*}{45} & C & 93.29 & 1.23 & 0.50 & 1.30 & 91.99 & 94.58 \\
\hline & $\mathrm{D}$ & 92.63 & 1.00 & 0.41 & 1.05 & 91.58 & 93.68 \\
\hline & $E$ & 98.49 & 4.32 & 1.76 & 4.54 & 93.96 & 103.03 \\
\hline & A & 93.06 & 1.51 & 0.62 & 1.58 & 91.48 & 94.65 \\
\hline & B & 92.41 & 0.77 & 0.31 & 0.81 & 91.60 & 93.21 \\
\hline \multirow[t]{5}{*}{60} & $C$ & 95.12 & 1.65 & 0.67 & 1.73 & 93.39 & 96.85 \\
\hline & $D$ & 92.72 & 0.77 & 0.32 & 0.81 & 91.91 & 93.53 \\
\hline & $E$ & 94.10 & 1.59 & 0.65 & 1.67 & 92.43 & 95.77 \\
\hline & A & 92.76 & 1.62 & 0.66 & 1.70 & 91.06 & 94.46 \\
\hline & B & 92.15 & 1.95 & 0.80 & 2.05 & 90.09 & 94.20 \\
\hline \multirow[t]{3}{*}{90} & $C$ & 94.16 & 0.87 & 0.35 & 0.91 & 93.25 & 95.07 \\
\hline & $D$ & 92.07 & 1.02 & 0.42 & 1.07 & 91.00 & 93.14 \\
\hline & $E$ & 94.88 & 1.45 & 0.59 & 1.53 & 93.36 & 96.41 \\
\hline
\end{tabular}

at different sampling times are shown in Table 7. MDT and VR are also called statistical moments, which can be used to establish relationships between in vitro and in vivo data (19) and to compare different dissolution profiles. The RD can be used to study the changes in the mechanism of drug release during the dissolution test by comparing the calculated values with those of theoretical physical models shown in Table 2 (19). The MDT values clearly show that all brands have very similar dissolution profiles before $30 \mathrm{~min}$. Because there is near complete release after 45 min, the MDT values of brands $A$ and $B$ show significant deficiencies compared with those of brands $C, D$, and $E$. The RD values of the different brands change with the dissolution process, indicating the variations of the release mechanism. Within $20 \mathrm{~min}$, all of the brands show zero- order release mechanisms as the calculated RD values are almost equal to the theoretical value of 0.3 . From 20 to 60 min, brands $A$ and $B$ show the constant Hixson-Crowell (cube-root) release mechanisms, whereas brand $C$ shows the change of release mechanism from Hixson-Crowell to Higuchi model (pseudo first-order) to first-order. Brand D shows only one change of release mechanism from Hixson-Crowell to Higuchi model; however, brand E shows a significant release mechanism during the dissolution process. The MDT values for the different brands are shown in Figure 3 , illustrating that brands $A$ and $B$ or brands $C$ and $D$ have very similar dissolution profiles, whereas brand $E$ is significantly different from the others.

Fit factors are important quantitative methods that have been recommended by the FDA guidelines for industry $(6$, 
Table 7. Mean Dissolution Time (MDT), Variance of Dissolution Time (VT), and Relative Dispersion (RD) Values for Different Brands

\begin{tabular}{|c|c|c|c|c|}
\hline Time (min) & Brand & MDT (min) & VT $\left(\min ^{2}\right)$ & RD \\
\hline \multirow{5}{*}{20} & A & 6.92 & 15.51 & 0.32 \\
\hline & B & 6.56 & 13.14 & 0.31 \\
\hline & C & 6.91 & 15.45 & 0.32 \\
\hline & D & 6.92 & 15.49 & 0.32 \\
\hline & E & 6.71 & 14.19 & 0.31 \\
\hline \multirow{5}{*}{30} & A & 8.05 & 33.66 & 0.52 \\
\hline & B & 7.69 & 31.98 & 0.54 \\
\hline & C & 8.61 & 41.82 & 0.56 \\
\hline & D & 8.69 & 42.89 & 0.57 \\
\hline & $E$ & 8.70 & 45.07 & 0.60 \\
\hline \multirow{5}{*}{45} & A & 8.33 & 41.69 & 0.60 \\
\hline & B & 8.02 & 41.25 & 0.64 \\
\hline & C & 9.80 & 73.12 & 0.76 \\
\hline & D & 10.06 & 78.41 & 0.78 \\
\hline & E & 10.58 & 92.60 & 0.83 \\
\hline \multirow{5}{*}{60} & A & 8.48 & 48.1 & 0.67 \\
\hline & B & 7.97 & 39.18 & 0.62 \\
\hline & C & 10.62 & 106.15 & 0.94 \\
\hline & D & 10.10 & 80.15 & 0.79 \\
\hline & $E$ & 8.62 & 11.04 & 0.15 \\
\hline \multirow{5}{*}{90} & A & 8.26 & 33.73 & 0.49 \\
\hline & B & 7.78 & 26.53 & 0.44 \\
\hline & C & 9.96 & 64.48 & 0.65 \\
\hline & D & 9.64 & 50.48 & 0.54 \\
\hline & $\mathrm{E}$ & 9.17 & 47.00 & 0.56 \\
\hline
\end{tabular}

20) for the comparison of dissolution profiles. Brand A was selected as the reference profile in this study. Results obtained from the tests are shown in Table 8 with the same observations found previously. The similarity factor $f_{2}$ is more sensitive in finding dissimilarity between dissolution curves than the difference factor $f_{1}$, and the values of fit factors are dependent on the number of sampling time points chosen (9-11). According to the FDA guidelines, $f_{1}$ values up to 15 and $f_{2}$ values greater than 50 should ensure equivalence of the dissolution curves, indicating an average difference of no more than $10 \%$ at the sample time points. Based on these guidelines, all five brands of commercially available aspirin tablets show dissolution curve equivalence. In addition, brand B is almost identical to the reference brand $A$.

\section{Model-Dependent Methods}

In this study, the various functions in Table 2 were fitted for dissolution times up to $45 \mathrm{~min}$ and $90 \mathrm{~min}$. At $45 \mathrm{~min}$,

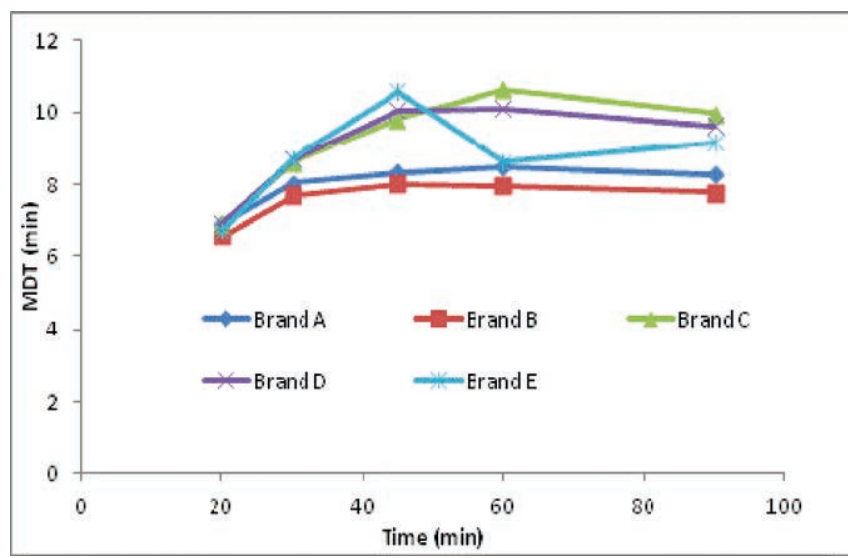

Figure 3. MDT values of the five brands of aspirin tablets at different cumulative time points.

Table 8. Fit Factors for the Five Brands of Aspirin Tablets Based on the Average of Six Tablets

\begin{tabular}{|c|c|c|c|}
\hline \multirow[b]{2}{*}{ Time (min) } & \multirow[b]{2}{*}{ Brand } & \multicolumn{2}{|c|}{ Fit Factor } \\
\hline & & $f_{1}$ & $f_{2}$ \\
\hline \multirow{4}{*}{10} & $A / B$ & 4.18 & 75.58 \\
\hline & $\mathrm{A} / \mathrm{C}$ & 5.78 & 69.11 \\
\hline & $A / D$ & 7.57 & 63.54 \\
\hline & $\mathrm{A} / \mathrm{E}$ & 2.26 & 86.45 \\
\hline \multirow{4}{*}{20} & $A / B$ & 2.03 & 81.95 \\
\hline & $\mathrm{A} / \mathrm{C}$ & 5.84 & 66.47 \\
\hline & $\mathrm{A} / \mathrm{D}$ & 7.58 & 61.02 \\
\hline & $A / E$ & 3.61 & 74.53 \\
\hline \multirow{4}{*}{30} & $A / B$ & 1.42 & 85.25 \\
\hline & $\mathrm{A} / \mathrm{C}$ & 4.64 & 69.31 \\
\hline & $A / D$ & 6.23 & 63.50 \\
\hline & $A / E$ & 2.37 & 78.39 \\
\hline \multirow{4}{*}{45} & $A / B$ & 1.10 & 87.43 \\
\hline & $\mathrm{A} / \mathrm{C}$ & 3.54 & 72.16 \\
\hline & $A / D$ & 4.57 & 66.49 \\
\hline & $\mathrm{A} / \mathrm{E}$ & 3.41 & 71.35 \\
\hline \multirow{4}{*}{60} & $A / B$ & 1.02 & 88.70 \\
\hline & $\mathrm{A} / \mathrm{C}$ & 3.25 & 73.54 \\
\hline & $\mathrm{A} / \mathrm{C}$ & 3.66 & 68.78 \\
\hline & $\mathrm{A} / \mathrm{C}$ & 2.92 & 73.38 \\
\hline \multirow{4}{*}{90} & $A / B$ & 0.96 & 89.67 \\
\hline & $\mathrm{A} / \mathrm{C}$ & 7.41 & 74.97 \\
\hline & $\mathrm{A} / \mathrm{C}$ & 3.15 & 70.58 \\
\hline & $\mathrm{A} / \mathrm{C}$ & 2.81 & 74.37 \\
\hline
\end{tabular}


Table 9. Model Parameters and Descriptive Statistics of Regression for 45 min

\begin{tabular}{|c|c|c|c|c|c|c|c|c|c|c|}
\hline Model & Brand & $C_{1}$ & $\mathrm{SD}_{1}$ & $C_{2}$ & $\mathrm{SD}_{2}$ & $C_{3}$ & $\mathrm{SD}_{3}$ & $r^{2}$ & $\boldsymbol{R}_{\max }$ & SRRMS \\
\hline & $A$ & $1.37 \times 10^{-1}$ & $1.27 \times 10^{-2}$ & 93.0 & $7.34 \times 10^{-1}$ & & & $9.97 \times 10^{-1}$ & $7.82 \times 10^{-1}$ & $4.82 \times 10^{-1}$ \\
\hline & B & $1.52 \times 10^{-1}$ & $7.58 \times 10^{-3}$ & 92.0 & $8.70 \times 10^{-1}$ & & & $9.84 \times 10^{-1}$ & 1.71 & $9.97 \times 10^{-1}$ \\
\hline \multirow[t]{5}{*}{ First-order } & $C$ & $1.19 \times 10^{-1}$ & $1.34 \times 10^{-2}$ & 92.4 & 1.82 & & & $9.75 \times 10^{-1}$ & 2.58 & 1.67 \\
\hline & D & $1.16 \times 10^{-1}$ & $1.07 \times 10^{-2}$ & 91.5 & 1.63 & & & $9.71 \times 10^{-1}$ & 2.78 & 1.85 \\
\hline & $\mathrm{E}$ & $1.13 \times 10^{-1}$ & $1.04 \times 10^{-2}$ & 96.4 & 2.61 & & & $9.35 \times 10^{-1}$ & 4.20 & 2.93 \\
\hline & A & 2.73 & $2.72 \times 10^{-2}$ & & & & & -9.96 & 42.3 & 30.8 \\
\hline & B & 2.73 & $3.62 \times 10^{-2}$ & & & & & -14.9 & 45.2 & 31.8 \\
\hline \multirow[t]{5}{*}{ Zero-order } & $C$ & 2.67 & $7.50 \times 10^{-2}$ & & & & & -5.80 & 38.8 & 27.8 \\
\hline & $\mathrm{D}$ & 2.64 & $4.51 \times 10^{-2}$ & & & & & -5.25 & 37.9 & 27.0 \\
\hline & $E$ & 2.78 & $5.60 \times 10^{-2}$ & & & & & -4.86 & 40.2 & 27.8 \\
\hline & A & 16.5 & $2.03 \times 10^{-1}$ & & & & & -1.26 & 17.8 & 14.0 \\
\hline & B & 16.5 & $2.36 \times 10^{-1}$ & & & & & -2.51 & 20.3 & 14.9 \\
\hline \multirow[t]{5}{*}{ Higuchi } & C & 16.1 & $5.10 \times 10^{-1}$ & & & & & $-1.35 \times 10^{-1}$ & 14.8 & 11.3 \\
\hline & $\mathrm{D}$ & 15.8 & $3.02 \times 10^{-1}$ & & & & & $1.00 \times 10^{-3}$ & 14.2 & 10.8 \\
\hline & $\mathrm{E}$ & 16.6 & $2.92 \times 10^{-1}$ & & & & & $1.14 \times 10^{-1}$ & 15.4 & 10.8 \\
\hline & A & $1.86 \times 10^{-1}$ & $2.62 \times 10^{-2}$ & 47.5 & 4.65 & & & $8.84 \times 10^{-1}$ & 3.50 & 31.6 \\
\hline & B & $1.61 \times 10^{-1}$ & $1.19 \times 10^{-2}$ & 51.7 & 2.72 & & & $9.98 \times 10^{-1}$ & 2.59 & 23.9 \\
\hline \multirow[t]{5}{*}{ Korsmeyer-Peppas } & $\mathrm{C}$ & $2.32 \times 10^{-1}$ & $3.42 \times 10^{-2}$ & 39.8 & 5.73 & & & $9.60 \times 10^{-1}$ & 2.40 & 21.3 \\
\hline & D & $2.39 \times 10^{-1}$ & $2.69 \times 10^{-2}$ & 38.2 & 3.89 & & & $9.68 \times 10^{-1}$ & 2.26 & 19.4 \\
\hline & $\mathrm{E}$ & $2.47 \times 10^{-1}$ & $2.96 \times 10^{-2}$ & 39.1 & 3.51 & & & $9.89 \times 10^{-1}$ & 1.78 & 1.20 \\
\hline & A & $3.12 \times 10^{-2}$ & $2.38 \times 10^{-4}$ & 91.5 & 1.38 & & & $7.52 \times 10^{-1}$ & 7.86 & 4.63 \\
\hline & B & $3.16 \times 10^{-2}$ & $2.49 \times 10^{-4}$ & 91.4 & 1.22 & & & $4.43 \times 10^{-1}$ & 10.4 & 5.95 \\
\hline \multirow[t]{5}{*}{ Hixson-Crowell } & $C$ & $3.20 \times 10^{-2}$ & $6.46 \times 10^{-4}$ & 88.0 & 3.67 & & & $9.06 \times 10^{-1}$ & 5.32 & 3.26 \\
\hline & $\mathrm{D}$ & $3.20 \times 10^{-2}$ & $8.07 \times 10^{-4}$ & 86.8 & 2.32 & & & $9.19 \times 10^{-1}$ & 4.80 & 3.07 \\
\hline & $\mathrm{E}$ & $3.28 \times 10^{-2}$ & $1.51 \times 10^{-3}$ & 90.0 & 1.27 & & & $9.01 \times 10^{-1}$ & 5.26 & 3.62 \\
\hline & A & 7.27 & $6.41 \times 10^{-1}$ & $9.39 \times 10^{-1}$ & $9.95 \times 10^{-2}$ & 93.7 & 1.47 & $9.99 \times 10^{-1}$ & $5.60 \times 10^{-1}$ & $3.56 \times 10^{-1}$ \\
\hline & $\mathrm{B}$ & 6.05 & $5.86 \times 10^{-1}$ & $7.86 \times 10^{-1}$ & $1.34 \times 10^{-1}$ & 93.9 & 1.82 & $9.73 \times 10^{-1}$ & 2.83 & 1.51 \\
\hline \multirow[t]{5}{*}{ Weibull } & $C$ & 9.59 & 2.44 & $7.16 \times 10^{-1}$ & $1.43 \times 10^{-1}$ & 99.7 & 5.01 & $9.98 \times 10^{-1}$ & $6.81 \times 10^{-1}$ & $4.58 \times 10^{-1}$ \\
\hline & D & 10.9 & 2.73 & $6.69 \times 10^{-1}$ & $1.97 \times 10^{-1}$ & 103 & 9.68 & $9.98 \times 10^{-1}$ & $6.67 \times 10^{-1}$ & $4.52 \times 10^{-1}$ \\
\hline & $\mathrm{E}$ & 206 & 306 & $5.38 \times 10^{-1}$ & $1.88 \times 10^{-1}$ & 168 & 99.4 & $9.97 \times 10^{-1}$ & $8.72 \times 10^{-1}$ & $6.06 \times 10^{-1}$ \\
\hline & A & -3.49 & $7.31 \times 10^{-1}$ & 4.48 & $9.24 \times 10^{-1}$ & 95.7 & 2.91 & $9.97 \times 10^{-1}$ & $8.57 \times 10^{-1}$ & $5.36 \times 10^{-1}$ \\
\hline & $B$ & -2.55 & $8.81 \times 10^{-1}$ & 3.67 & $9.42 \times 10^{-1}$ & 96.3 & 2.94 & $9.95 \times 10^{-1}$ & $9.19 \times 10^{-1}$ & $5.78 \times 10^{-1}$ \\
\hline \multirow[t]{3}{*}{ Logistic } & $C$ & -2.22 & $6.94 \times 10^{-1}$ & 2.68 & 1.05 & 109 & 11.4 & $9.97 \times 10^{-1}$ & $8.69 \times 10^{-1}$ & $5.63 \times 10^{-1}$ \\
\hline & $\mathrm{D}$ & -2.07 & $7.13 \times 10^{-1}$ & 2.35 & 1.04 & 115 & 19.1 & $9.97 \times 10^{-1}$ & $8.31 \times 10^{-1}$ & $5.42 \times 10^{-1}$ \\
\hline & $E$ & -2.24 & $7.46 \times 10^{-1}$ & 1.68 & $8.93 \times 10^{-1}$ & 352 & 367 & $9.97 \times 10^{-1}$ & $9.44 \times 10^{-1}$ & $6.37 \times 10^{-1}$ \\
\hline
\end{tabular}

dissolution of all of the aspirin tablets was greater than $90 \%$ and approached the plateau. The dissolutions for $90 \mathrm{~min}$ represent the whole set of points determined. The parameters of the mathematical models are shown in Tables 9 and 10. The descriptive statistics of regression for the dissolution data in Tables 9 and 10 were calculated based on the average mean dissolution data curve, including determination coefficient $\left(r^{2}\right)$, maximum residual in absolute size between fitted and actual percentages of drug dissolved, $R_{\max }$ and the square root of mean of residuals square (SRRMS). The selection of the best fitting model is based on the descriptive statistics of the regression in the following order: higher determination coefficient, smaller maximum residual and smaller the square root of mean of residuals square.

For the dissolution curves up to $45 \mathrm{~min}$, the best function for the different brands overall is the logistic model 
Table 10. Model Parameters and Descriptive Statistics of Regression for 90 min

\begin{tabular}{|c|c|c|c|c|c|c|c|c|c|c|}
\hline Model & Brand & $C_{1}$ & $S_{1}$ & $C_{2}$ & $S_{2}$ & $C_{3}$ & $\mathrm{SD}_{3}$ & $r^{2}$ & $R_{\max }$ & SRRMS \\
\hline \multirow{5}{*}{ First-order } & A & $1.38 \times 10^{-1}$ & $1.40 \times 10^{-2}$ & 93.0 & 1.02 & & & $9.98 \times 10^{-1}$ & $7.87 \times 10^{-1}$ & $4.05 \times 10^{-1}$ \\
\hline & B & $1.51 \times 10^{-1}$ & $7.87 \times 10^{-3}$ & 92.1 & $9.63 \times 10^{-1}$ & & & $9.87 \times 10^{-1}$ & 1.78 & $8.28 \times 10^{-1}$ \\
\hline & $C$ & $1.13 \times 10^{-1}$ & $1.45 \times 10^{-2}$ & 93.8 & 1.38 & & & $9.75 \times 10^{-1}$ & 2.86 & 1.66 \\
\hline & $D$ & $1.14 \times 10^{-1}$ & $1.04 \times 10^{-2}$ & 92.1 & 1.10 & & & $9.77 \times 10^{-1}$ & 2.89 & 1.57 \\
\hline & $\mathrm{E}$ & $1.17 \times 10^{-1}$ & $7.63 \times 10^{-3}$ & 95.3 & 1.59 & & & $9.43 \times 10^{-1}$ & 4.01 & 2.48 \\
\hline \multirow{5}{*}{ Zero-order } & A & 1.54 & $1.69 \times 10^{-2}$ & & & & & -24.1 & 55.3 & 42.3 \\
\hline & B & 1.53 & $1.97 \times 10^{-2}$ & & & & & -34.2 & 57.2 & 42.9 \\
\hline & $C$ & 1.54 & $2.64 \times 10^{-2}$ & & & & & -13.4 & 50.2 & 39.7 \\
\hline & $\mathrm{D}$ & 1.51 & $1.60 \times 10^{-2}$ & & & & & -13.5 & 49.3 & 39.2 \\
\hline & $\mathrm{E}$ & 1.57 & $2.32 \times 10^{-2}$ & & & & & -14.9 & 52.3 & 41.3 \\
\hline \multirow{5}{*}{ Higuchi } & A & 13.1 & $1.34 \times 10^{-1}$ & & & & & -6.12 & 31.2 & 22.5 \\
\hline & B & 13.0 & $1.68 \times 10^{-1}$ & & & & & -9.32 & 31.6 & 23.2 \\
\hline & $C$ & 13.0 & $2.72 \times 10^{-1}$ & & & & & -2.62 & 29.2 & 19.9 \\
\hline & $\mathrm{D}$ & 12.8 & $1.56 \times 10^{-1}$ & & & & & -2.69 & 29.1 & 19.8 \\
\hline & $\mathrm{E}$ & 13.2 & $1.87 \times 10^{-1}$ & & & & & -3.18 & 30.7 & 21.2 \\
\hline \multirow{5}{*}{$\begin{array}{l}\text { Korsmeyer- } \\
\text { Peppas }\end{array}$} & A & $1.12 \times 10^{-1}$ & $1.88 \times 10^{-2}$ & 59.1 & 4.26 & & & $7.23 \times 10^{-1}$ & 6.71 & 4.44 \\
\hline & $\mathrm{B}$ & $9.69 \times 10^{-2}$ & $9.13 \times 10^{-3}$ & 62.4 & 2.59 & & & $7.36 \times 10^{-1}$ & 5.39 & 3.71 \\
\hline & $C$ & $1.53 \times 10^{-1}$ & $2.60 \times 10^{-2}$ & 50.5 & 5.98 & & & $8.35 \times 10^{-1}$ & 5.89 & 4.24 \\
\hline & D & $1.50 \times 10^{-1}$ & $1.88 \times 10^{-2}$ & 50.0 & 3.97 & & & $8.11 \times 10^{-1}$ & 6.09 & 4.47 \\
\hline & $\mathrm{E}$ & $1.40 \times 10^{-1}$ & $1.26 \times 10^{-2}$ & 53.7 & 2.45 & & & $7.60 \times 10^{-1}$ & 7.00 & 5.08 \\
\hline \multirow{5}{*}{$\begin{array}{l}\text { Hixson- } \\
\text { Crowell }\end{array}$} & A & $1.66 \times 10^{-2}$ & $1.08 \times 10^{-4}$ & 98.5 & $8.72 \times 10^{-1}$ & & & -2.45 & 28.3 & 15.7 \\
\hline & B & $1.66 \times 10^{-2}$ & $9.30 \times 10^{-5}$ & 98.2 & 1.10 & & & $-4.32 \times 10^{-1}$ & 31.2 & 16.7 \\
\hline & $\mathrm{C}$ & $1.64 \times 10^{-2}$ & $7.58 \times 10^{-5}$ & 98.2 & 2.09 & & & $-5.60 \times 10^{-1}$ & 24.7 & 13.1 \\
\hline & D & $1.64 \times 10^{-2}$ & $4.83 \times 10^{-5}$ & 96.5 & 1.19 & & & $-5.70 \times 10^{-1}$ & 24.2 & 12.9 \\
\hline & $\mathrm{E}$ & $1.64 \times 10^{-2}$ & $1.09 \times 10^{-4}$ & 100 & 1.60 & & & $-8.10 \times 10^{-1}$ & 26.3 & 14.0 \\
\hline \multirow{5}{*}{ Weibull } & A & 7.25 & $5.92 \times 10^{-1}$ & $9.70 \times 10^{-1}$ & $7.31 \times 10^{-2}$ & 93.1 & 1.18 & $9.98 \times 10^{-1}$ & $6.42 \times 10^{-1}$ & $3.41 \times 10^{-1}$ \\
\hline & B & 6.10 & $5.54 \times 10^{-1}$ & $8.51 \times 10^{-1}$ & $9.19 \times 10^{-2}$ & 92.7 & 1.18 & $9.77 \times 10^{-1}$ & 2.77 & 1.29 \\
\hline & $C$ & 8.50 & 1.14 & $7.94 \times 10^{-1}$ & $6.91 \times 10^{-2}$ & 95.4 & $9.90 \times 10^{-1}$ & $9.95 \times 10^{-1}$ & 1.01 & $7.12 \times 10^{-1}$ \\
\hline & $\mathrm{D}$ & 8.42 & $9.61 \times 10^{-1}$ & $8.21 \times 10^{-1}$ & $1.04 \times 10^{-1}$ & 93.3 & $7.89 \times 10^{-1}$ & $9.92 \times 10^{-1}$ & 1.36 & $9.29 \times 10^{-1}$ \\
\hline & $\mathrm{E}$ & 8.05 & $6.36 \times 10^{-1}$ & $8.21 \times 10^{-1}$ & $5.44 \times 10^{-2}$ & 96.3 & 1.91 & $9.58 \times 10^{-1}$ & 3.82 & 2.12 \\
\hline \multirow{5}{*}{ Logistic } & A & -4.09 & $6.46 \times 10^{-1}$ & 5.15 & $8.10 \times 10^{-1}$ & 93.8 & 1.52 & $9.94 \times 10^{-1}$ & 1.08 & $6.29 \times 10^{-1}$ \\
\hline & B & -3.36 & $6.73 \times 10^{-1}$ & 4.60 & $6.85 \times 10^{-1}$ & 93.4 & 1.40 & $9.89 \times 10^{-1}$ & 1.19 & $7.61 \times 10^{-1}$ \\
\hline & $C$ & -2.77 & $4.96 \times 10^{-1}$ & 3.46 & $6.92 \times 10^{-1}$ & 98.0 & 1.78 & $9.89 \times 10^{-1}$ & 1.51 & 1.08 \\
\hline & $\mathrm{D}$ & -2.88 & $6.42 \times 10^{-1}$ & 3.59 & $6.74 \times 10^{-1}$ & 95.5 & 1.35 & $9.83 \times 10^{-1}$ & 1.85 & 1.33 \\
\hline & $E$ & -2.84 & $3.27 \times 10^{-1}$ & 3.62 & $3.60 \times 10^{-1}$ & 98.3 & 2.38 & $9.44 \times 10^{-1}$ & 4.38 & 2.45 \\
\hline
\end{tabular}

followed by the Weibull model with almost identical results. As both the logistic and Weibull models are empirical, there is no direct connection with release mechanism. From the results, the first-order model provides a good fit for all of the brands, indicating similar drug release mechanisms for aspirin drugs under different brands. A slightly different conclusion was drawn based on the model-independent approaches shown in Table 7 in which the RDs of brands A and $B$ are around the theoretical value 0.6 of the cube-root release mechanism. For the dissolution curves up to $90 \mathrm{~min}$, the best-fit function is the Weibull model followed by the logistic model. The first-order model presents regression results similar to those of the Weibull and logistic models, which indicates the diffusion controlled-release mechanism for all of the brands of aspirin tablets. We have to stress that the above conclusions are drawn based on purely 
Table 11. Comparison of the Model Parameters of Test Brands Against the Reference Brand by Extended t-Test at Risk $\alpha=0.05$

\begin{tabular}{|c|c|c|c|c|c|c|c|c|c|c|c|c|c|c|c|c|}
\hline \multirow[b]{3}{*}{ Brand } & \multicolumn{8}{|c|}{ Up to $45 \mathrm{~min}$} & \multicolumn{8}{|c|}{ Up to $90 \mathrm{~min}$} \\
\hline & \multicolumn{3}{|c|}{ Logistic } & \multicolumn{3}{|c|}{ Weibull } & \multicolumn{2}{|c|}{ First-order } & \multicolumn{3}{|c|}{ Logistic } & \multicolumn{3}{|c|}{ Weibull } & \multicolumn{2}{|c|}{ First-order } \\
\hline & $C_{1}$ & $C_{2}$ & Conclusions & $C_{1}$ & $C_{2}$ & Conclusions & $C_{1}$ & Conclusions & $C_{1}$ & $C_{2}$ & Conclusions & $C_{1}$ & $C_{2}$ & Conclusions & $C_{1}$ & Conclusion \\
\hline B & 2.01 & 0.015 & Similar & 3.42 & 2.24 & Different & 2.44 & Different & 1.91 & 0.113 & Similar & 3.47 & 2.49 & Different & 2.08 & Similar \\
\hline C & 3.11 & 0.073 & $\begin{array}{l}\text { Different (1 } \\
\text { parameter) }\end{array}$ & 2.26 & 3.12 & Different & 2.51 & Different & 3.97 & 0.051 & $\begin{array}{l}\text { Different (1 } \\
\text { parameter) }\end{array}$ & 2.38 & 4.29 & Different & 2.94 & Different \\
\hline D & 3.43 & 0.060 & $\begin{array}{l}\text { Different (1 } \\
\text { parameter) }\end{array}$ & 3.21 & 2.98 & Different & 3.18 & Different & 3.25 & 0.072 & $\begin{array}{l}\text { Different (1 } \\
\text { parameter) }\end{array}$ & 2.53 & 2.88 & Different & 3.37 & Different \\
\hline$E$ & 2.93 & 0.018 & $\begin{array}{l}\text { Different (1 } \\
\text { parameter) }\end{array}$ & 1.59 & 4.62 & $\begin{array}{l}\text { Different (1 } \\
\text { parameter) }\end{array}$ & 3.57 & Different & 4.23 & 0.184 & $\begin{array}{l}\text { Different (1 } \\
\text { parameter) }\end{array}$ & 2.26 & 4.00 & Different & 3.11 & Different \\
\hline
\end{tabular}

mathematical analysis, some of which could be erroneous or meaningless. For example, if all tablets dissolved completely near $45 \mathrm{~min}$, it would be inappropriate to draw any conclusions by the comparison.

The model parameters of the logistic, Weibull, and first-order models for the test brands $B, C, D$, and $E$ were compared against reference brand $A$ using a two-variable $t$-test described above and summarized in Table 11. It is concluded that brand $B$ is similar to reference brand $A$ for both $45 \mathrm{~min}$ and $90 \mathrm{~min}$ if the logistic model is selected and one of the parameters of the logistic model for brands $C, D$ and $E$ is different from that of the reference $A$. For the first-order model, brand $B$ is similar to brand A for 90 min, whereas there is a slight difference between the two brands for $45 \mathrm{~min}$. The parameters of the first-order model of the brands $C, D$, and $E$ are different from that of the reference $A$ for both 45 and $90 \mathrm{~min}$, but the differences are marginal. If the Weibull model is selected, it can been seen that all of the brands are different at both 45 and 90 min because the Weibull function is based on an empirical model. Generally, these results are in agreement with the previous findings of the model-independent approaches.

\section{CONCLUSION}

Quality control tests including mechanical hardness, friability, disintegration, and in vitro dissolution were carried out to evaluate the performance characteristics of five brands of commercially available aspirin tablets. Brands A and B or brands C, D, and E have similar hardness, friability, and disintegration characteristics, indicating that the two groups of the brands have different manufacturing conditions. Comparisons of dissolution profiles of the different brands were carried out by both model-independent and model-dependent approaches. All brands of aspirin tablets have a similar first-order drug release mechanism based on the model-dependent approach; furthermore, the model-dependent approach can also differentiate between the different brands, concluding that brands $A$ and $B$ are similar in good agreement with the results of the mechanical and disintegration tests. Different model-independent approaches were used to compare the dissolution profiles including ratio tests and fit factors. The ratio tests of MDT, VT, and $\mathrm{RD}$ can translate a dissolution curve into a single value and they can also infer the drug release mechanisms of the formulations. However, it would be difficult to identify the difference between the dissolution curves that depend on the final time point considered and difference limit values chosen. Fit factors using a difference and similarity approach have been recommended by the FDA and are simple and easy to implement. In the FDA guidelines, clear limit values of up to 15 for $f_{1}$ and greater than 50 for $f_{2}$ indicate the equivalence of the dissolution profiles. Based on this, all five brands of commercially available aspirin tablets are considered similar. Finally, we would like to emphasize that all of the above conclusions were drawn based on the dissolution tests at 75 rpm; different conclusions could probably be obtained if a lower rotation speed was selected in the tests. In the meantime, it is incomplete that any conclusion regarding the mechanism of drug lease was drawn based on purely mathematical analysis, which could be erroneous or meaningless.

\section{REFERENCES}

1. Aspirin Foundation. Uses of Aspirin. http://www. aspirin-foundation.com/uses/index.html (accessed Jan 18, 2013).

2. MedicineNet. Drug class and mechanism of aspirin. http://www.medicinenet.com/acetylsalicylic_acid/ article.htm (accessed Jan 18, 2013).

3. Thun, M. J.; Namboodiri, M. M.; Calle, E. E.; Flanders, W. D.; Heath, C. W. Jr. Aspirin Use and Risk of Fatal Cancer. Cancer Res. 1993, 53 (6), 1322-1327.

4. Brown, C. K.; Chokshi, H. P.; Nickerson, B.; Reed, R. A. Acceptable Analytical Practices for Dissolution Testing of Poorly Soluble Compounds. Pharm. Technol. 2004, 28 (12), 56-65.

5. Maggio, R. M.; Castellano, P. M.; Kaufman, T. S. A new principal component analysis-based approach for testing "similarity" of drug dissolution profiles. Eur. J. Pharm. Sci. 2008, 34 (1), 66-77.

6. Dissolution Testing of Immediate Release Solid Oral Dosage Forms; Guidance for Industry; U.S. Department 
of Health and Human Services, Food and Drug Administration, Center for Drug Evaluation and Research (CDER), U.S. Government Printing Office: Washington, DC, 1997.

7. O'Hara, T.; Dunne, A.; Butler, J.; Devane, J. A review of methods used to compare dissolution profile data. Pharm. Sci. Technol. Today 1998, 1 (5), 214-223.

8. Yuksel, N.; Kanik, A. E.; Baykara, T. Comparison of in vitro dissolution profiles by ANOVA-based, modeldependent and -independent methods. Int. J. Pharm. 2000, 209 (1-2), 57-67.

9. Polli, J. E.; Rekhi, G. S.; Augsburger, L. L.; Shah, V. P. Methods to compare dissolution profiles and a rationale for wide dissolution specifications for metoprolol tartrate tablets. J. Pharm. Sci. 1997, 86 (6), 690-700.

10. Costa, F. O.; Sousa, J. J. S.; Pais, A. A. C. C.; Formosinho, S. J. Comparison of dissolution profiles of Ibuprofen pellets. J. Control. Release 2003, 89 (2), 199-212.

11. Costa, P. An alternative method to the evaluation of similarity factor in dissolution testing. Int. J. Pharm. 2001, $220(1-2), 77-83$.

12. Sathe, P. M.; Tsong, Y.; Shah, V. P. In-vitro dissolution profile comparison: statistics and analysis, model dependent approach. Pharm. Res. 1996, 13 (12), 17991803.

13. Adams, E.; Coomans, D.; Smeyers-Verbeke, J.; Massart, D. L. Application of linear mixed effects models to the evaluation of dissolution profiles. Int. J. Pharm. 2001, $226(1-2), 107-125$.

14. Adams, E.; Coomans, D.; Smeyers-Verbeke, J.; Massart, D. L. Non-linear mixed effects models for the evaluation of dissolution profiles. Int. J. Pharm. 2002, 240 (1-2), 37-53.

15. Pillay, V.; Fassihi, R. Evaluation and comparison of dissolution data derived from different modified release dosage forms: an alternative method. J. Control. Release 1998, 55 (1), 45-55.

16. Shah, V. P.; Tsong, Y.; Sathe, P.; Liu, J.-P. In Vitro Dissolution Profile Comparison-Statistics and Analysis of the Similarity Factor, $f_{2}$. Pharm. Res. 1998, 15 (6), 889-896.

17. Moore, J. W.; Flanner, H. H. Mathematical Comparison of Dissolution Profiles. Pharm. Tech. 1996, 20 (6), 64-74.

18. Pinto, J. F.; Podczeck, F.; Newton, J. M. The use of statistical moment analysis to elucidate the mechanism of release of a model drug from pellets produced by extrusion and spheronisation. Chem. Pharm. Bull. 1997, 45 (1), 171-180.

19. Ocaña, J.; Frutos, G.; Sánchez, P. Using the similarity factor $f_{2}$ in practice: A critical revision and suggestions for its standard error estimation. Chemometr. Intell. Lab. 2009, 99 (1), 49-56.

20. SUPAC-MR: Modified Release Solid Oral Dosage Forms, Scale-Up and Postapproval Changes: Chemistry, Manufacturing, and Controls; In Vitro Dissolution Testing and In Vivo Bioequivalence Documentation; Guidance for Industry; U.S. Department of Health and Human Services, Food and Drug Administration, Center for Drug Evaluation and Research (CDER), U.S. Government Printing Office: Washington, DC, 1997.

21. Costa, P.; Sousa Lobo, J. M. Modeling and comparison of dissolution profiles. Eur. J. Pharm. Sci. 2001, 13 (2), 123-133.

22. The British Pharmacopoeia; The Stationary Office: London, 2011.

23. Aulton's Pharmaceutics: The Design and Manufacture of Medicines, 3rd ed.; Aulton, M. E., Ed.; Churchill Livingstone: London, 2007. 